Revista de Derecho

Universidad Católica del Norte

Sección: Ensayos

Ańo 18 - No 1, 2011

pp. 213-239

\title{
EL MECANISMO DE SOLUCIÓN DE DIFERENCIAS DE LA OMC. UN ELEMENTO DE SEGURIDAD Y PREVISIBILIDAD EN EL SISTEMA MULTILATERAL DE COMERCIO*
}

\section{CRISTIÁN DELPIANO LIRA*}

RESUMEN: Uno de los objetivos fundamentales del sistema de solución de diferencias de la Organización Mundial de Comercio consiste en otorgar seguridad y previsibilidad al sistema multilateral de comercio. El presente artículo pretende otorgar una visión panorámica acerca de la forma en que se lleva a cabo este objetivo, a través de un análisis orgánico y procesal de las actuaciones ante los Grupos Especiales y el Órgano de Apelación. De esta manera, el artículo identificará las materias fundamentales a través de las cuales el sistema funciona, así como los criterios del Órgano de Solución de Diferencias que han permitido su desarrollo desde 1995 hasta la fecha. El autor examina de esta manera el Órgano de Solución de Diferencias y los órganos que le sirven de soporte (Grupos Especiales y Órgano de Apelación), el fundamento de las reclamaciones, y los actores del sistema.

PALABRAS CLAVE: Organización Mundial de Comercio (OMC) solución de diferencias - sistema multilateral de comercio - política comercial - GATT.

\section{A DISPUTE SETTLEMENT BODY OF THE WTO. AN ELEMENT OF SECURITY AND PREVISIBILITY IN THE MULTILATERAL TRADING SYSTEM}

ABSTRACT: One of the main objectives of the dispute settlement body of the World Trade Organization is to provide security and previsibility to the multilateral trading system. This article intends to give

El presente trabajo se enmarca dentro de la ejecución del proyecto DGIP 10301273 financiado por la Universidad Católica del Norte. El contenido del artículo es una versión parcial del módulo de Solución de Diferencias dictado por el suscrito en la Universidad de Tarapacá, en el marco del curso de "Introducción al Sistema Multilateral de Comercio", impartido por la Cátedra OMC del Instituto de Estudios Internacionales de la Universidad de Chile y la Organización Mundial de Comercio.

Fecha de recepción: 13 de enero de 2011.

Fecha de aceptación: 15 de abril de 2011.

** Abogado, DEA en Derecho Internacional Universidad de Salamanca (España), profesor de Derecho Internacional Público de la Escuela de Derecho de la Universidad Católica del Norte, sede Antofagasta. Correo electrónico: codelpiano@ucn.cl 
an overview about how this aim is carried out through the organic and procedural analysis of the proceedings before the panel and the Appellate Body. Thus, this article tries to identify the key areas through which the system carries out its objective and the criteria of the Dispute Settlement Body that have enabled its development from 1995 to nowadays. The author examines in this way the Dispute Settlement Body and bodies to support them (Panel and Appellate Body), the merits of complaints, and the actors of the system.

KEY WORDS: World Trade Organization (WTO) - dispute settlement - Multilateral Trade System - commercial politics - General Agreement on Tariffs and Trade (GATT).

\section{INTRODUCCIÓN}

El mejor de los acuerdos internacionales no funciona adecuadamente si sus obligaciones no pueden hacerse valer cuando una de sus partes falla en el cumplimiento de tales obligaciones. Con esta acertada frase comienza el Manual relativo al Sistema de Solución de Diferencias (en adelante "SSD") de la Organización Mundial de Comercio (en adelante "OMC") 1 .

Esta premisa ha sido debidamente tomada en cuenta durante las negociaciones de la Ronda Uruguay de Negociaciones Comerciales, de la cual ha resultado un importante reforzamiento del Mecanismo de Solución de Diferencias (en adelante "MSD") de la OMC.

En efecto, la regulación acerca de la solución de diferencias resultante de esta ronda de negociaciones se plasmó en el Entendimiento Relativo a las Normas y Procedimientos por el que se Rige la Solución de Diferencias de la OMC (en adelante "ESD"), que se caracteriza por la obligatoriedad para todos los Estados miembros de la OMC. Ello debido a que todos los acuerdos resultantes de la Ronda Uruguay deben ser aceptados en su conjunto, salvo los acuerdos plurilaterales del anexo 4 del Acuerdo por el que se Establece la $\mathrm{OMC}^{2}$.

World Trade Organization (2004). A Handbook on the WTO Dispute Settlement System, Cambridge University Press, p. 1.

2 A diferencia de la situación que se produjo como resultado de la Ronda Tokio de Negociaciones comerciales, en la que se dio un cierto fenómeno de fragmentación del sistema GATT, debido a que los Estados podían aceptar ciertos acuerdos, denominados códigos, y rechazar otros. 
Este artículo tiene por objeto identificar ciertos aspectos orgánicos y procesales del MSD que permiten otorgar seguridad y previsibilidad al sistema multilateral de comercio, y que constituyen un parámetro de actuación para los Estados miembros de la OMC. De esta manera, si bien las decisiones del OSD solo tienen valor para las partes en conflicto, se presentan como un parámetro ineludible del comportamiento futuro de los Estados en el sistema multilateral de comercio.

Así, el artículo se divide en cinco apartados. El primero tiene por objeto realizar un breve repaso histórico en relación con los aspectos fundamentales del rudimentario sistema creado en el GATT de 1947, y su posterior reforzamiento en el ESD, con el consiguiente impacto en el sistema multilateral de comercio.

El segundo apartado se dedica a un análisis institucional que permite el adecuado funcionamiento del sistema, a través de los Grupos Especiales, el Órgano de Apelación y el OSD. Abordaremos también el alcance de la jurisdicción y sus límites.

En el tercer apartado, analizaremos las funciones, objetivos y propósitos fundamentales del sistema, así como la aplicación que ha recibido por parte de los Grupos Especiales y del Órgano de Apelación. El cuarto y quinto apartado tienen por objeto analizar la base jurídica sobre las cuales se puede recurrir al SSD. De esta manera, abordaremos las denominadas reclamaciones por infracción de normas, así como las reclamaciones sin infracción, que probablemente constituya una de las grandes características diferenciadoras del sistema.

El quinto y último apartado se dedica a quienes pueden recurrir y concurrir al sistema, con especial referencia a las denominadas comunicaciones Amicus Curiae. Terminaremos con unas breves reflexiones finales.

\section{1) DesarRollo histórico DEL MSD}

Como sabemos, el MSD de la OMC es heredero del antiguo MSD creado con ocasión del GATT de 1947, así como de toda su práctica posterior. Esta práctica fue primeramente codificada durante la Ronda de Tokio, y luego reforzada en la Ronda Uruguay.

La base sobre la cual se estructuró el MSD del GATT de 1947 la encontramos en los artículos XXII y XXIII del Acuerdo General, que contempla una etapa de consultas, y una segunda etapa "cuasijudicial".

\section{(1.1.) EL MSD EN EL GATT DE 1947}

Los artículos XXII y XXIII del GATT de 1947 contienen algunas reglas relativamente simples y de poca eficacia para resolver las controversias entre las Partes Contratantes, que contemplaban un primer recurso a las 
consultas directas entre los Estados implicados en la diferencia, además de un recurso a la reunión de las partes contratantes, considerado el único órgano establecido para la gestión del acuerdo ${ }^{3}$.

En lo fundamental proveía tres clases de soluciones, las decisiones, recomendaciones y suspensión de obligaciones ${ }^{4}$. Las Partes Contratantes debían realizar una "encuesta" sobre la cuestión y establecer recomendaciones. Además, se facultaba para permitir la suspensión de concesiones o de otras obligaciones dimanantes del Acuerdo General5.

Es discutible si en realidad los artículos XXII y XXIII contenían efectivamente un sistema para la solución de las diferencias en el GATT ${ }^{6}$. Sin embargo, el desarrollo posterior de estas normas nos permite afirmar que ya no se trataba de un conjunto de normas aisladas, sino que un sistema, que aunque rudimentario, fue mejorando a través de la práctica de los Estados en la aplicación de los artículos XXII y XXIII, coincidente con el propio desarrollo del GATT.

En una primera etapa, las diferencias se resolvieron por el propio Consejo del GATT, para pasar a la constitución de los nacientes Grupos de Trabajo. Estos grupos estaban compuestos por representantes de todas las partes contratantes interesadas, quienes adoptaban la decisión por consenso. Con posterioridad, los Grupos de Trabajo fueron reemplazados por Grupos Especiales o panels de tres a cinco miembros independientes y no relacionados con las partes en el litigio. Su labor fundamental consistía en emitir un informe con recomendaciones para la solución de la diferencia, para remitirla al Consejo del GATT. Una vez que el Consejo lo aprobaba por consenso pasaba a ser vinculante para los Estados en la diferencia. De esta forma, los panels fueron construyendo un acervo jurisprudencial de una importancia fundamental para los criterios que actualmente adopta el $\mathrm{OSD}^{7}$. Sin embargo, su defecto de origen consistía en el

3 Véase Bou Franch (2005). Nuevas Controversias Internacionales y Nuevos Mecanismos de Solución, Valencia, Tirant lo Blanch, p. 286.

4 Petersmann, E.-U. (1997). International Trade Law and the GATT/WTO Dispute Settlement System, London: Kluwer Law International, p. 38.

5 Sin embargo, y a pesar del amplio poder de las partes contratantes para suspender las concesiones, fue utilizado solo una vez durante los 48 años de vigencia del GATT. "In 1952, the Netherlands was authorized to take retaliatory trade restrictions vis-à-vis the US in response to a persistent infringement by that country of article XI" (Petersmann (1997) 46.

6 En este sentido véase Diez-Eochleitner, J. (2005). "La Unión Europea ante la Reforma del Sistema de Solución de Diferencias de la OMC", en De Faramiñan, J. M. (coord.): Globalización y Comercio Internacional: Actas de la XX Jornadas de la Asociación Española de Profesores de Derecho Internacional y Relaciones Internacionales, Madrid, Asociación Española de Profesores de Derecho internacional y Relaciones Internacionales - BOE, 2005 , p. 62.

7 Petersmann señala que esta práctica ha derivado hacia una orientación del sistema hacia las normas, más que hacia consideraciones políticas (Petersmann (1997) 48. La relevancia de los criterios adoptados por los panels en el GATT ha sido reconocida por el Órgano de Apelación en el asunto caso Japón - Bebidas Alcohólicas, en el que rescató la importancia que 
hecho que en la decisión debía participar también la parte perdedora en la diferencia, lo que otorgaba una cierta inestabilidad a los informes de los panels. A pesar de ello, era usual que los informes fueran finalmente adoptados por las Partes Contratantes, salvo en seis ocasiones ${ }^{8}$, básicamente frente a resoluciones que pretendían que la parte demandada reembolsara a la parte vencedora los perjuicios producidos por el acto considerado contrario a la normativa GATT. Cabe agregar a ello, que las partes contratantes demandadas en forma individual mayoritariamente se abstenían del bloqueo de las decisiones por consenso y admitían a trámite las disputas en las cuales se encontraban envueltos, aun cuando fuera en su propio detrimento en el corto plazo. Ello debido al interés sistémico a largo plazo que tenían y el conocimiento que un excesivo uso del derecho de veto podía resultar en una respuesta similar por los otros. De acuerdo a esto, los panels eran establecidos y sus reportes frecuentemente adoptados, aunque muchas veces con retraso (aunque la autorización de contramedidas solo fue concedida una vez).

De esta forma, la fragilidad del sistema creado en el GATT de 1947 dio pie para que el mecanismo se fuera desarrollando por la vía de la práctica subsiguiente, que lo convirtió "en el más utilizado de todas las organizaciones internacionales existentes").

La práctica en materia de solución de diferencias en el GATT se configuró por dos grandes vías. La primera de ellas se refiere a la práctica desarrollada en el seno de los Grupos Especiales, que en muchas ocasiones se han visto plasmadas en los propios informes que han remitido para la

tienen los informes adoptados de los Grupos Especiales del GATT para los criterios futuros del MSD de la OMC, aun cuando no sean obligatorios sino para solucionar la diferencia específica entre las partes en litigio. A mayor abundamiento, también señaló la importancia que tienen los informes no adoptados del GATT. En este sentido, dichos informes carecen de valor normativo en el sistema del GATT o de la OMC, puesto que no han sido avalados por decisiones de las Partes Contratantes del GATT o de los Miembros de la OMC. Sin embargo, "pueden contener útiles orientaciones en el razonamiento seguido en un informe no adoptado de un grupo especial que a su juicio fuera pertinente al asunto que examinaba" (Japón - Impuestos sobre las Bebidas Alcohólicas [Japón - Bebidas Alcohólicas], WT/DS8/ $\mathrm{AB} / \mathrm{R}$, Informe del Órgano de Apelación de 4 de octubre de 1996). Un Grupo Especial fue más allá todavía. En efecto, en el asunto Argentina - Calzados se basó en el informe no adoptado del Grupo Especial del GATT relativo a Comunidades Europeas - Bananos II (Véase en este sentido Argentina - Medidas que afectan a las Importaciones de Calzados, Textiles, Prendas de Vestir y otros Artículos (Argentina - Calzados), WT/DS56/AB/R, Informe del Órgano de Apelación de 27 de marzo de 1998, párrafo 43.

8 El profesor Petersmann cita los casos EC - Member's states import regimes for bananas (DS32/R); EC - Import regime for bananas (DS38/R); USA - Restrictions on imports of tuna (DS21/R and DS29/R); USA - Taxes on automobiles (DS31/R) y Canada - Measures affecting sale of gold coins (L/5863) (Petersmann (1997) 74).

9 Montañ́ I Mora, M. (1997). La OMC y el Reforzamiento del Sistema GATT, Madrid, McGraw-Hill, p. 47. 
aprobación de las Partes Contratantes ${ }^{10}$. La segunda vía se manifiesta en los acuerdos que han adoptado las Partes Contratantes, que por una parte han sistematizado las prácticas de los panels ya señalados, y por otra, han modificado prácticas procesales emergentes en materia de solución de controversias. Diversos documentos han manifestado estas cuestiones, entre las cuales se cuentan la decisión de 5 de abril de $1966^{11}$, el Entendimiento Relativo a las Notificaciones, las Consultas, la Solución de Diferencias y la Vigilancia de 1979, la Decisión relativa a la solución de diferencias contenida en la Declaración Ministerial de 1982 y la Decisión sobre Solución de Diferencias de 30 de noviembre de $1984^{12}$.

A pesar de dicha evolución, hubo muchas otras cuestiones que se mantuvieron intactas durante toda la vigencia del GATT y hasta la conclusión de la Ronda Uruguay, siendo la más importante de ellas la necesidad que las instancias del proceso fueran aprobadas por consenso en el seno del Consejo del GATT. De esta manera, la decisión de someter la disputa a un panel, los informes de los mismos, así como las contramedidas autorizadas en contra de la parte contratante que no implementara las recomendaciones y resoluciones del Consejo General, debían ser

10 Los informes y las prácticas que han venido desarrollando los panels han reflejado también ciertos principios procesales de actuación, tales como el principio de economía procesal, bilateralidad de la audiencia, la delimitación de su ámbito de competencias, la carga de la prueba, el principio de confidencialidad, la presunción de anulación o menoscabo, entre otros Nichols, P. (1996). "GATT Doctrine", Virginia Journal of International Law, vol. 36, No 2, p. 379; Mcgovern, E. (1996): "Dispute Settlement in the GATT - Adjudication or Negotiation?", en Hilf, M., Jacobs, F. and Petersmann, E.-U. (eds.): The European Community and GATT, Deventer: Kluwer Law and Taxation Publishers, p. 78, Mitchell, A. (2008): Legal Principles in WTO Disputes, Cambridge University Press, 2008, especialmente pp. 145 y ss.; Hudec, R. (1990). The GATT Legal System and World Trade Diploma$c y$, New Hampshire Butterworth Legal Publishers, pp. 63 y ss.).

11 La decisión de 1966 estuvo marcada por la aparición de los países en desarrollo y menos desarrollados provenientes del proceso de descolonización de Âfrica y Asia, lo que obligó a las partes contratantes a disponer de un procedimiento diferenciado entre partes desarrolladas y en vías de desarrollo. Entre estos, se dispuso la intervención del Director General del GATT en la cuestión debatida, acudiendo con sus buenos oficios a intentar una solución satisfactoria a la diferencia. En segundo lugar, se previó la obligación de las partes contratantes de estudiar las medidas ante el incumplimiento de la parte desarrollada en la diferencia (véase los apartados 8 y 10 de la decisión, IBDD S14/20).

12 World Trade Organization (2004) 13. Adicional a ello, antes de la Ronda Tokio de Negociaciones comerciales se realizaron una serie de enmiendas a los artículos XXII y XXIII del GATT, entre las cuales se contó la facultad explícita de las Partes Contratantes para intervenir en las consultas si las partes en la disputa no eran capaces de solucionar la controversia por sí mismas, la autorización de las Partes Contratantes para suspender concesiones y otras medidas de retorsión cuando las circunstancias sean lo suficientemente graves, la posibilidad de asociar a terceros Estados a las consultas que se formulen de acuerdo al artículo XXII. Véase Stewart, T. (ed.) (1993). The GATT Uruguay Round. A Negotiating History (1986-1992), The Hague, Kluwer Law International, pp. 2675 y ss.; también puede verse Petersmann, E. - Tersmannon "The Dispute Settlement System of the World Trade Organization and the Evolution of the GATT Dispute Settlement System Since 1948”, Common Market Law Review, vol. 31, N 6, pp. 1157 y ss. 
aprobadas por consenso positivo de las Partes Contratantes, incluida la perdedora en el caso de los informes de los panels. De esta manera, las Partes Contratantes gozaban de un derecho individual de veto sobre las resoluciones del Consejo del GATT, que caracteriza al sistema como políticamente orientado.

\section{(1.2.) LOS CAMBIOS FUNDAMENTALES PRODUCIDOS EN LA RONDA URUGUAY}

Como parte de los resultados de la Ronda Uruguay de Negociaciones Comerciales, el ESD introdujo un reforzamiento significativo al SSD. Entre otros factores, estableció procedimientos más detallados para todas las instancias de la diferencia, incluidos plazos específicos. Por otra parte, estableció el Órgano de Apelación, que tiene la facultad de revisar la interpretación jurídica de los informes de los Grupos Especiales (Art. 17.6 del ESD), otorgándole una cierta continuidad y uniformidad a los criterios que adopte el OSD. De esta manera, tanto Grupos Especiales como el Órgano de Apelación han construido una jurisprudencia que permite clarificar las reglas del juego, incrementando de esta forma la seguridad jurídica, y la calidad del precedente GATT/OMC ${ }^{13}$.

Entre otras modificaciones, dispuso plazos estrictos para las actuaciones de los Grupos Especiales, el Órgano de Apelación, e incluso para el propio OSD, consagró un tratamiento especial y diferenciado para los países en desarrollo que participen del $\mathrm{SSD}^{14}$, y estableció un marco integrado aplicable a todos los acuerdos abarcados.

Sin duda, la innovación más importante del ESD consistió en la eliminación del derecho de las partes de bloquear la constitución de los Grupos Especiales, así como la adopción de los informes de estos y del Órgano de Apelación, salvo que exista consenso para no establecer el Grupo Especial (Art. 6.1 del ESD), o para no adoptar el informe (Arts. 16.4 y 17.14 del ESD), cuestión que se suele denominar la regla del "consenso negativo" o derecho colectivo de veto ${ }^{15}$. Esta innovación ha hecho que la adopción de los informes por parte del OSD se constituya como un mero ritual de formalidad en la $\mathrm{OMC}^{16}$, caracterizándolo como un sistema orientado hacia las normas.

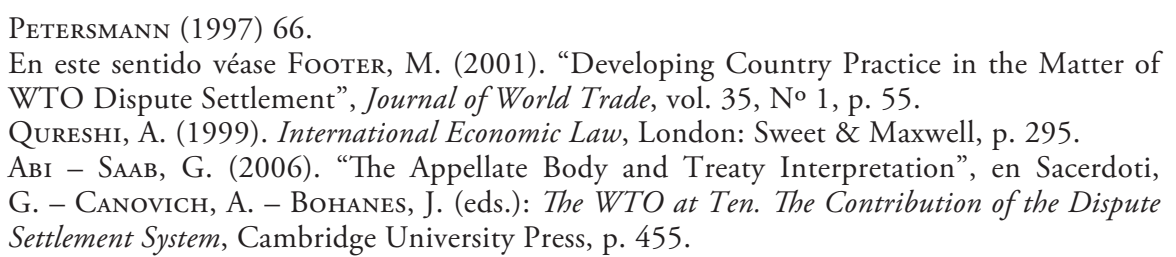


En todo caso, este esquema de toma de decisiones es excepcional y solamente aplicable a la adopción de los informes al interior del MSD y otras cuestiones expresamente señaladas en el ESD, dado que la regla general sigue siendo el consenso positivo ${ }^{17}$. Con este nuevo sistema se resta todo valor por sí mismo al rechazo de los informes por la parte perdedora. Junto con ello, también se elimina el incentivo de los países más poderosos de recurrir a medidas unilaterales para promover sus intereses comerciales ${ }^{18}$, configurando un mecanismo que, asumiendo los principios básicos y el acervo generado por varias décadas de aplicación de los artículos XXII y XXIII del GATT, incorpora diversos elementos tendientes a reforzar su carácter jurisdiccional ${ }^{19}$.

Por último, cabe mencionar que el artículo 23 del ESD obliga a los Estados miembros a recurrir al MSD cuando se trate de "reparar el incumplimiento de obligaciones u otro tipo de anulación o menoscabo de las ventajas resultantes de los acuerdos abarcados, o de un impedimento al logro de cualquiera de los objetivos de los acuerdos abarcados, (...)", obligándolos, a su vez, a seguir las normas establecidas en dicha norma para las reclamaciones y las medidas de retorsión ${ }^{20}$.

De esta manera, se "explicita la voluntad de los miembros de no adoptar medidas unilaterales en defensa de sus políticas comerciales y, en consecuencia, de seguir una aproximación institucionalizada en la resolución de los conflictos comerciales" 21 .

\section{2) ÓRGANOS INVOLUCRADOS EN LA SOLUCIÓN DE CONTROVERSIAS}

Desde el punto de vista operativo, el proceso completo de solución de diferencias involucra en primer término a las partes y terceros en la

\footnotetext{
17 Así se desprende del artículo 2.4 del Entendimiento de 1994, que establece que "En los casos que las normas y procedimientos del presente Entendimiento establezcan que el OSD debe adoptar una decisión, se procederá por consenso", entendiéndose por tal que ningún miembro presente en la reunión del OSD en que se adopte la decisión se oponga formalmente a ella.

18 Rodríguez, M. (2005). "La Solución de Controversias en la OMC. Evolución y Reforma (1995-2004)", en De Faramiñan, J. M. (coord.): Globalización y Comercio Internacional: Actas de la XX Jornadas de la Asociación Española de Profesores de Derecho Internacional y Relaciones Internacionales, Madrid, Asociación Española de Profesores de Derecho internacional y Relaciones Internacionales - BOE, p. 46.

19 González, L. N. (1998). Política Comercial y Relaciones Exteriores de la Unión Europea, Madrid, Tecnos, p. 163.

20 Un interesante análisis del artículo 23 en relación con los mecanismos de solución de diferencias existentes en los acuerdos comerciales regionales puede verse en el asunto Méjico - Medidas Fiscales sobre los Refrescos y otras Bebidas (Méjico - Refrescos), WT/DS308/ AB/R, Informe del Órgano de Apelación de 6 de marzo de 2006.

21 Diez-Eochleitner (2005) 65.
} 
controversia ${ }^{22}$. Además, se puede identificar la actuación de otras instituciones como el OSD, los Grupos Especiales, el Órgano de Apelación, la Secretaría de la OMC, árbitros, y expertos independientes. Entre estas instituciones, se pueden identificar algunas independientes y cuasijudiciales, como los Grupos Especiales, el Órgano de Apelación y los árbitros, así como otras de índole política, como es el caso del OSD. En el presente artículo solo abordaremos los tres primeros.

\section{(2.1) EL OSD}

El artículo IV.3 del Acuerdo por el que se Establece la OMC dispone que "El Consejo General se reunirá según proceda para desempeñar las funciones del Órgano de Solución de Diferencias establecido en el Entendimiento sobre Solución de Diferencias". De esta manera el Consejo General adopta la calidad de OSD, compuesto en consecuencia por todos los Estados miembros de la OMC, a través de delegaciones gubernamentales (normalmente delegaciones diplomáticas), que a la vez representan las posiciones de sus propios Estados dentro del Consejo y del OSD. Esencialmente, tanto el Consejo como el OSD tienen el mismo ámbito de actuación, pero operando en diferentes capacidades ${ }^{23}$.

Como funciones fundamentales se cuenta la designación de los Grupos Especiales, adopción de los informes, tanto de los panels como del Órgano de Apelación, la vigilancia en la aplicación de las resoluciones y recomendaciones y la autorización para suspender concesiones y otras obligaciones en el marco de los acuerdos abarcados ${ }^{24}$.

De conformidad con el artículo 2.4 del ESD, el OSD adopta sus decisiones por "consenso positivo", entendiéndose por tal el hecho que ninguno de los miembros presentes en la reunión se oponga formalmente a ella $^{25}$, de manera que la delegación que pretenda oponerse a una decisión del OSD, deberá estar presente en la reunión en que dicha decisión se adopte, y manifestar en términos formales y explícitos su oposición. Sin embargo, el ESD contempla cuatro casos en que la regla del consenso positivo (o derecho individual de veto) se invierte, adoptando sus decisiones según el denominado "consenso negativo". Estos casos son respecto de la decisión de establecimiento de un Grupo Especial ${ }^{26}$ y la adopción de sus informes ${ }^{27}$, la adopción de los informes del Órgano de Apelación ${ }^{28}$, y la

\footnotetext{
22 Por tercero se entiende todo miembro que tenga un interés sustancial en un asunto sometido a un Grupo Especial y así lo haya notificado al OSD (Art. 10.2 del ESD).

QURESHI (1999) 290.

Artículo 2.1 del ESD.

Nota 1 al artículo 2.4 .

Artículo 6.1 del ESD.

Artículo 16.4 del ESD.

Artículo 17.14 del ESD.
} 
autorización para la suspensión de concesiones u otras obligaciones, dentro del marco de la vigilancia en la implementación de las resoluciones y recomendaciones ${ }^{29}$.

De esta manera, las decisiones que debe adoptar por consenso positivo son la aprobación de las personas que integrarán la lista indicativa de la cual puedan elegirse los integrantes de los Grupos Especiales ${ }^{30}$, el nombramiento de los miembros del Órgano de Apelación ${ }^{31}$, y la aceptación del plazo propuesto por la parte afectada para aplicar las resoluciones y recomendaciones del $\mathrm{OSD}^{32}$.

\section{(2.2.) LOS GRUPOS ESPECIALES Y EL ÓRGANO DE APELACión}

Los Grupos Especiales tienen como función principal asistir al OSD a cumplir las funciones que le incumben en virtud del ESD y de los acuerdos abarcados. Para satisfacer este cometido, debe hacer una evaluación objetiva del asunto sometido a su consideración. Ello incluye una apreciación de los hechos, de la aplicabilidad de los acuerdos involucrados y su conformidad con estos, así como la formulación de otras conclusiones que ayuden al OSD a hacer las recomendaciones o dictar las resoluciones previstas en los acuerdos respectivos ${ }^{33}$.

Su composición proviene de una lista indicativa que debe mantener la Secretaría de personas que hayan sido propuestas por cualesquiera de los Estados miembros y aprobada por el OSD ${ }^{34}$.

Los Grupos Especiales son formados por tres personas, salvo acuerdo de las partes, quienes pueden convenir que el Grupo Especial lo formen cinco. Son nombrados a propuesta de la Secretaría de la OMC, con acuerdo de las partes, quienes no podrán oponerse, salvo "razones imperiosas"35. En caso de desacuerdo, su composición será establecida por el Director General, en consulta con el Presidente del OSD y con el Presidente del Consejo o Comité correspondiente.

Artículo 22.6 y 22.7 del ESD.

Artículo 8.4 del ESD.

Artículo 17.2 del ESD.

Artículo 21.3 a) del ESD.

Artículo 11 del ESD.

Artículo 8.4 del ESD. Por su parte, el artículo 8.1 establece que "estarán formados por personas muy competentes, funcionarios gubernamentales o no, a saber, personas que anteriormente hayan integrado un grupo especial o hayan presentado un alegato en él, hayan actuado como representantes de un Miembro o de una parte contratante del GATT de 1947 o como representantes en el Consejo o Comité de cualquier acuerdo abarcado o del respectivo acuerdo precedente o hayan formado parte de la Secretaría del GATT, hayan realizado una actividad docente o publicado trabajos sobre derecho mercantil internacional o política comercial internacional, o hayan ocupado un alto cargo en la esfera de la política comercial en un Miembro".

35 Artículo 8.6 del ESD. 
Los miembros de los Grupos Especiales deben actuar siempre a título personal, garantizando de esta manera su independencia e imparcialidad. En consecuencia, se les prohíbe a los Estados miembros darles instrucciones y ejercer sobre ellos cualquier clase de influencia con respecto a los asuntos sometidos al Grupo Especial ${ }^{36}$.

A diferencia de los Grupos Especiales, el Órgano de Apelación es permanente, siendo integrado por siete personas, quienes actúan por turno de tres en cada caso. Sus miembros son nombrados por un período de cuatro años cada uno, y renovable por una sola vez ${ }^{37}$. Tiene como función revisar las cuestiones de derecho tratadas en el informe del Grupo Especial y las interpretaciones jurídicas formuladas por este, en el caso que alguno de los Estados parte de la diferencia haya apelado de la decisión del Grupo Especial ${ }^{38}$.

\section{(2.3.) JURISDiCCIÓN DE LOS PANELS Y DEL ÓRGANO DE APELACIÓN}

De conformidad con el artículo 1.1 del ESD, la jurisdicción del OSD se extiende a todas las disposiciones de consultas y solución de diferencias de los acuerdos abarcados. De esta manera, todas las diferencias que puedan producirse en el marco de los acuerdos OMC se encuentran sujetas a las normas del ESD.

Sin embargo, esta jurisdicción no es absoluta. En efecto, una primera limitación se encuentra en el artículo 1.2, que dispone que estas normas se aplican "sin perjuicio de las normas y procedimientos especiales o adicionales que en materia de solución de diferencias contienen los acuerdos abarcados", de manera que frente a un conflicto entre las normas del ESD y las normas y procedimientos especiales del apéndice 2 al ESD, prevalecerán estas últimas ${ }^{39}$.

Artículo 8.7 del ESD.

Está integrado por personas de reconocido prestigio, con competencia técnica acreditada en derecho, en comercio internacional y en la temática de los acuerdos abarcados en general. No están vinculados a ningún gobierno, y serán representativos en términos generales de la composición de la OMC (Artículo 17.3 del ESD).

38 Se excluye de la apelación a los terceros Estados (Artículo 17.4 del ESD).

39 Así lo ha reafirmado a partir de las normas y procedimientos del Acuerdo de Subvenciones y Medidas Compensatorias y el Acuerdo Antidumping (véase los asuntos Comunidades Europeas - Subvenciones a la Exportación de Azúcar, WT/DS265/AB/R, WT/DS266/AB/R y WT/ DS283/AB/R, Informe del Órgano de Apelación de 28 de abril de 2005, párrafo 333, así como también el asunto Estados Unidos - Examen por Extinción de los Derechos Antidumping sobre los Productos Planos de Acero al Carbono Resistentes a la Corrosión procedentes del Japón (Estados Unidos: Examen por Extinción: Acero resistente a la Corrosión), WT/DS244/AB/R, Informe del Órgano de Apelación de 15 de diciembre de 2003, nota 82 al párrafo 83). 
En segundo lugar, el OSD no tiene facultades para interpretar con carácter general y abstracto las normas de los acuerdos OMC, debido a que el propio acuerdo por el que es establece la OMC reserva esta facultad al Consejo General. El Órgano de Apelación ha sostenido que el proceso no se concibe para "alentar a los Grupos Especiales o al Örgano de Apelación a 'legislar' mediante la aclaración de disposiciones vigentes del Acuerdo sobre la $O M C$, fuera del contexto de la solución de una determinada diferencia. Un grupo especial solo necesita tratar las alegaciones que se deben abordar para resolver el asunto debatido en la diferencia" ${ }^{40}$. Sin embargo, el Grupo Especial podría examinar una cuestión a pesar que ello no se traduzca en resoluciones y recomendaciones $^{41}$.

\section{3) FUNCIONES Y OBJETIVOS DEL MSD}

Los propósitos fundamentales del SSD se encuentran expresados en el artículo 3.2 del ESD, que señala que:

"El sistema de solución de diferencias de la OMC es un elemento esencial para aportar seguridad y previsibilidad al sistema multilateral de comercio. Los Miembros reconocen que este sistema sirve para preservar los derechos y obligaciones de los Miembros en el marco de los acuerdos abarcados y para aclarar las disposiciones vigentes de dichos acuerdos de conformidad con las normas usuales de interpretación de derecho internacional público" 42 .

De una manera funcional a estos propósitos, el artículo 3.7 dispone que:

"El objetivo del mecanismo de solución de diferencias es hallar una solución positiva a las diferencias. Se debe dar siempre preferencia a una solución mutuamente aceptable para las partes en la diferencia y que esté en conformidad con los acuerdos abarcados. De no llegarse a una solución de mutuo acuerdo, el primer objetivo del mecanismo

40 Estados Unidos - Medida que Afecta a las Importaciones de Camisas y Blusas de Tejidos de Lana Procedentes de la India (Estados Unidos - Camisas y Blusas), WT/DS33/AB/R, Informe del Órgano de Apelación de 25 de abril de 1997 (cursiva en el original). De esta manera, un Grupo Especial puede no considerar la compatibilidad de una medida con los acuerdos OMC (Argentina - Calzados, párrafo 6.15).

41 Estados Unidos - Subvenciones al Algodón Americano (Upland) (Estados Unidos - Algodón Americano (Upland)), WT/DS267/AB/R, Informe del Órgano de Apelación de 3 de marzo de 2005, párrafo 510.

42 Cursivas ańadidas. 
de solución de diferencias será en general conseguir la supresión de las medidas de que se trate si se constata que éstas son incompatibles con las disposiciones de cualquiera de los acuerdos abarcados" 43 .

De esta manera, se pueden identificar tres grandes propósitos del sistema. Por una parte, la aportación de seguridad y previsibilidad, en el entendido que el comercio de bienes y servicios no es conducido generalmente por los Estados, sino más bien por privados, lo que justifica que estos deban saber adecuadamente las normas y las conductas que deben seguir en su inserción en el comercio internacional, tanto en relación con la regulación en general, así como también la regulación específica que se aplica en su sector de actividad, especialmente cuando se trata de transacciones de largo plazo.

Teniendo esto presente, el ESD se propone proveer de un mecanismo rápido, eficiente, confiable y orientado hacia las normas, que permita resolver las disputas en torno a la interpretación y aplicación de las disposiciones de los acuerdos abarcados.

Además, se propone establecer un procedimiento que funcione adecuadamente ante el incumplimiento en la implementación de las medidas exigidas para adecuar la política comercial interna al sistema multilateral de comercio, de forma que en esta hipótesis el sistema permita establecer sanciones comerciales.

El segundo propósito del SSD consiste en preservar los derechos y obligaciones de los Estados miembros, de manera que ante una medida de política comercial que se estime inconsistente con las obligaciones provenientes de los Acuerdos OMC, el Estado que se sienta agraviado se encuentra facultado para invocar las normas y procedimientos del SSD, de manera de impugnar dicha medida.

Si las partes no logran una solución mutuamente convenida, a los Estados miembros envueltos en la controversia se les garantiza un procedimiento basado en normas y bajo los parámetros del debido proceso, en el que un órgano independiente e imparcial revisará los méritos de la reclamación y de la defensa. En consecuencia, el objetivo primordial del sistema no consiste en dictar sentencias ni tampoco desarrollar jurisprudencia, sino que solo proveer una solución a la diferencia presentada, preferiblemente a través de una solución mutuamente aceptable, cuestión que puede conseguirse en cualquier etapa de todo el procedimiento ${ }^{44}$.

De conformidad con el artículo 3.7 del ESD, si los Grupos Especiales o el Órgano de Apelación aceptan la pretensión de la parte reclamante, el objetivo será conseguir la supresión de la medida considerada inconsis-

Cursivas añadidas.

World Trade Organization (2004) 6. 
tente. De esta manera, el mismo artículo 3.7 dispone que la posibilidad de suspender en forma discriminatoria la aplicación de concesiones o el cumplimiento de otras obligaciones en el marco de los acuerdos abarcados será utilizado como último recurso, y siempre que el OSD autorice la adopción de estas medidas ${ }^{45}$.

Como tercer propósito, el sistema pretende aclarar las disposiciones vigentes de los acuerdos abarcados de conformidad con las normas usuales de interpretación del derecho internacional público ${ }^{46}$. Las normas de la $\mathrm{OMC}$, como cualquier precepto jurídico, requieren de la interpretación de un criterio general para un caso en particular. Este ejercicio a ratos resulta extremadamente difícil, especialmente teniendo en cuenta que muchas veces las normas adolecen de falta de claridad como resultado de ser la expresión de compromisos adquiridos en diversas rondas de negociaciones comerciales. De esta manera, los participantes de dichos procesos de negociación acuerdan un texto conciliatorio de diversas posiciones, que puede ser interpretado de más de una forma, de manera de satisfacer a las

45 De esta manera, el Derecho de la OMC contiene diversas particularidades en relación con el sistema general de responsabilidad internacional del Estado, cuestión que excede ampliamente el objeto de este trabajo. Sobre el particular véase Fernández, X. (2006). La OMC y el Derecho Internacional, Madrid: Marcial Pons, especialmente pp. 259 y ss. A mayor abundamiento, el SSD no define las responsabilidades legales de una parte contratante que haya violado sus obligaciones. Sin embargo, desde la práctica en materia de solución de diferencias del GATT se ha reconocido que "el objetivo de las Partes Contratantes ha sido siempre encontrar una solución positiva a las diferencias. Evidentemente, es preferible hallar una solución mutuamente aceptable para las partes en la diferencia. Si no se llega a una solución mutuamente aceptada, el objetivo primordial que suelen perseguir las PARTES Contratantes es conseguir la supresión de las medidas de que se trate si se constata que éstas son incompatibles con el Acuerdo General. No se debe conceder una compensación sino en el caso de que no sea factible suprimir inmediatamente las medidas incompatibles con el Acuerdo General y como solución provisional hasta su supresión. El último recurso previsto en el artículo XXIII para el país que se acoja a este procedimiento es la posibilidad de suspender la aplicación de concesiones o el cumplimiento de otras obligaciones de manera discriminatoria respecto de la otra parte contratante, siempre que las PARTEs ContraTANTES autoricen la adopción de estas medidas" (párrafo 4 del anexo "Exposición Acordada de la Práctica Consuetudinaria del GATT en Materia de Solución de Diferencias”, en el Entendimiento Relativo a las Notificaciones, las Consultas, la Solución de Diferencias y la Vigilancia, adoptado el 28 de noviembre de 1979 (Documento L/4907), como resultado de la Ronda de Tokio de Negociaciones Comerciales).

46 Esta interpretación se entiende sin perjuicio de la disposición del artículo IX.2 del Acuerdo por el que se Establece la OMC, que dispone que "La Conferencia Ministerial y el Consejo General tendrán la facultad exclusiva de adoptar interpretaciones del presente Acuerdo y de los Acuerdos Comerciales Multilaterales". Esta facultad exclusiva de la Conferencia Ministerial y del Consejo General debe entenderse como la posibilidad de adoptar interpretaciones imperativas de validez general para todos los miembros de la OMC, a diferencia de la interpretación que realizan los Grupos Especiales y el Órgano de Apelación, que solo obligan a las partes en la diferencia y circunscrito al tema específico sobre el que verse la disputa (World Trade Organization (2004) 4). Esta interpretación, en todo caso es aceptable sin perjuicio de la imposibilidad de negar la influencia que ejercen los informes sobre las conductas futuras de los Estados miembros de la OMC. 
diversas posturas ${ }^{47}$. En este sentido, se puede sostener incluso que el propio ejercicio de interpretación de los acuerdos OMC se configura como una extensión de la negociación original ${ }^{48}$.

De esta manera, el ESD reconoce la necesidad de aclarar los preceptos de la OMC a través de las normas consuetudinarias de interpretación, contenidas fundamentalmente en los artículos 31, 32 y 33 de la Convención de Viena sobre el Derecho de los Tratados de 1969 (en adelante "CVDT"), disposiciones de amplia aplicación en los informes de los Grupos Especiales y del Órgano de Apelación ${ }^{49}$.

En este sentido, el Órgano de Apelación ha agregado como corolario de las normas de la CVDT el principio del efecto útil de la interpretación, de manera que debe dar sentido y ha de afectar a todos los términos del tratado ${ }^{50}$. Cabe consignar que este principio fue excluido de la codificación por la Comisión de Derecho Internacional, de manera que no aparece dentro de aquellos establecidos en los artículos 31, 32 y 33 de la Convención de Viena sobre el Derecho de los Tratados, sin perjuicio de lo cual ha sido también ampliamente aplicado por los Grupos Especiales y el Órgano de Apelación ${ }^{51}$.

47 World Trade Organization (2004) 3.

48 El análisis de esta cuestión supera ampliamente los objetivos de este trabajo. Sin embargo, sobre el particular puede verse Qureshi (1999) 293 y ss.; Pauwelyn, J. (2001). "The Role of Public International Law in the WTO: How far can we go?", American Journal of International Law, vol. 95, pp. 535 y ss.

49 Los Grupos Especiales y el Órgano de Apelación han reiterado y seguido religiosamente estos criterios, dejándose guiar en sus razonamientos por sentencias e informes de otros tribunales internacionales, particularmente por la Corte Internacional de Justicia. Oesch, M. (2005). Standards of Review in WTO Dispute Resolution, Oxford University Press, p. 43. Algunos autores han sostenido, sin embargo, que en la interpretación y aplicación de estas normas se ha otorgado un estrecho margen de deferencia a las autoridades nacionales en la interpretación y aplicación de las normas $\mathrm{OMC}$, lo que puede explicarse por una autoridad y legitimidad del MSD que todavía es relativamente débil. Оеsch (2005) 44. También puede verse JACKson, J. H. (2000). The Jurisprudence of GATT \& the WTO, Cambridge University Press, 2000, pp. 133 y ss.

50 Estados Unidos - Pautas para la Gasolina Reformulada y Convencional (Estados Unidos - Gasolina), WT/DS2/AB/R, Informe del Órgano de Apelación de 29 de abril de 1996; Japón - Bebidas Alcohólicas. El artículo 31.1 de la CVDT dispone que "Un tratado deberá interpretarse de buena fe conforme al sentido corriente que haya de atribuirse a los términos del tratado en el contexto de éstos teniendo en cuenta su objeto y fin". En general, los Grupos Especiales suelen interpretar los acuerdos de la OMC enfocado más en el sentido gramatical y en el contexto, más que en el objeto y fin de estos (World Trade Organization (2004) 5). Por su parte, el artículo 32 dispone que los trabajos preparatorios tienen solo un rol subsidiario en materia de interpretación, pudiendo aplicarse exclusivamente cuando se vaya a confirmar la interpretación lograda bajo los métodos del artículo 31, o cuando la interpretación conforme a estos métodos deje ambiguo u obscuro el sentido o que conduzca a un resultado manifiestamente absurdo o irrazonable.

51 Véase en este sentido Van Damme, I. (2009). Treaty Interpretation by the WTO Appellate Body, Oxford University Press, pp. 275 y ss.; Аві - IAAB (2006) 462. 
Por último, el sistema impide la adopción de medidas unilaterales frente al incumplimiento de obligaciones u otro tipo de anulación o menoscabo de las ventajas resultantes de los acuerdos abarcados. En efecto, el artículo 23 del ESD dispone que, en ese caso, los miembros se encuentran obligados a recurrir a las normas y procedimientos del propio ESD. Como efecto de lo anterior, el artículo 23 tiene como efecto excluir otros foros para la solución de controversias relativas a las normas de la $\mathrm{OMC}^{52}$, así como impedir que los miembros determinen "que se ha producido una infracción, se han anulado o menoscabado ventajas o se ha comprometido el cumplimiento de uno de los objetivos de los acuerdos abarcados" $"$.

\section{4) BASE JURÍdica de LA RECLAMACIÓN}

El ESD conserva la base proveniente del GATT de 1947 en la materia, en la cual se contenían las normas originales sobre consultas y solución de diferencias. Estas normas se encuentran en los artículos XXII y XXIII que contienen un margen de actuación de los Grupos Especiales inédito en el Derecho internacional.

En efecto, el artículo XXIII establece que:

“1. En caso de que una parte contratante considere que una ventaja resultante para ella directa o indirectamente del presente Acuerdo se halle anulada o menoscabada o que el cumplimiento de uno de los objetivos del Acuerdo se halle comprometido a consecuencia de:

a) que otra parte contratante no cumpla con las obligaciones contraídas en virtud del presente Acuerdo; o

b) que otra parte contratante aplique una medida, contraria o no a las disposiciones del presente Acuerdo; o

c) que exista otra situación,..."

De esta manera, el concepto fundamental que subyace a cualquier controversia es el de anulación o menoscabo, que puede provenir de una violación de normas del GATT, de la aplicación de una medida que, sin ser contraria a los acuerdos OMC generen este efecto, que se denomina reclamación sin infracción, y por último de la existencia de otra situación ${ }^{54}$.

Méjico - Refrescos, párrafo 58.

Artículo 23.2 a) del ESD.

La existencia de "otra situación" de conformidad con el artículo XXIII.1 letra c) del GATT

fue entendida originalmente para ser utilizada en situaciones de emergencia económica, tales como crisis económicas generales, altos niveles de desempleo, colapso de precios de 


\section{(4.1.) LAS RECLAMACIONES POR VIOLACIÓN DE NORMAS}

La parte agraviada tendrá acceso al OSD cuando considere que una ventaja resultante de los acuerdos abarcados por el ESD ha sido anulada o menoscabada o que el cumplimiento de uno de los objetivos de estos acuerdos se halle comprometido como consecuencia de la violación de alguna de las disposiciones de los acuerdos de la OMC. Desde luego, esta hipótesis cubre la mayor parte de las reclamaciones que se han sucedido en el GATT, y actualmente en la OMC.

En este caso, el artículo 3.8 del ESD señala que la anulación o menoscabo se presume. La norma proviene de la práctica anterior al ESD, que ya había hecho de esta una presunción irrefutable ${ }^{55}$.

\section{(4.2.) LA RECLAMACIONES SIN INFRACCIÓN}

La segunda hipótesis permite al Estado miembro a recurrir al OSD cuando un Estado miembro considere que una medida, sin ser contraria a las normas de la OMC, anule o menoscabe una ventaja resultante de los acuerdos abarcados, o que el cumplimiento de uno de los objetivos de los acuerdos se halle comprometido.

commodities, etc. Cabe mencionar que en los más de sesenta ańos de existencia del MSD, nunca un Grupo Especial ha conocido una diferencia relativa al artículo XXIII.1 letra c). Sin embargo, esta posibilidad se consagró en el artículo 26.2 del ESD, en el cual hace aplicable la regla del consenso negativo hasta el momento de las actuaciones en que el informe del Grupo Especial se distribuya entre los miembros, siendo aplicable a las actuaciones posteriores las normas y procedimientos de solución de diferencias contenidos en la decisión de 12 de abril de 1989 a la consideración de las recomendaciones y para su adopción y a la vigilancia y aplicación de dichas resoluciones y recomendaciones. De esta manera, se establece la característica adicional que los informes deben ser adoptados por consenso positivo, presentándose como una reminiscencia del procedimiento del GATT (véase entre otros World Trade Organization (2004) 34; Matsushita, M. - Tchoenbaum, T. - HavroiDIS, P. (2006): The World Trade Organization. Law, Practice and Policy, Oxford University Press, p. 122; Palmeter, D. - Lavroidis, P. (2004): Dispute Settlement in the World Trade Organization. Practice and Procedure, Cambridge University Press, p. 164).

55 Esta disposición ya constaba en el párrafo 5 del anexo sobre la Exposición acordada de la práctica consuetudinaria del GATT en materia de solución de diferencias, resultante del Entendimiento Relativo a las Notificaciones, las Consultas, la Solución de Diferencias y la Vigilancia, del 28 de noviembre de 1979, que señalaba, en lo pertinente, que "En los casos de contravención de las obligaciones contraídas en virtud del Acuerdo General, se presume que la medida constituye un caso de anulación o menoscabo. La presunción de anulación o menoscabo de un beneficio entrañará ipso facto la necesidad de dilucidar si las circunstancias son suficientemente graves para que esté justificada la autorización de suspender concesiones u obligaciones, si así lo solicita la parte contratante que presente la reclamación. Esto significa que normalmente se presume que la infracción de las reglas tiene efectos perjudiciales para otras partes contratantes, y en estos casos la impugnación del cargo corresponde a la parte contratante contra la que se ha presentado la reclamación" (cursiva en el original). Véase también GATT Analytical Index, pp. 659 y ss. 
De esta manera, frente a una reclamación sin infracción el Estado reclamante no tiene la necesidad de acreditar la violación de alguna norma de la OMC, aunque sí deberá acreditar la anulación o menoscabo de las ventajas resultantes de los acuerdos.

En efecto, el artículo 26.1 a) del ESD establece que "la parte reclamante apoyará con una justificación detallada cualquier reclamación relativa a una medida que no esté en contradicción con el acuerdo abarcado pertinente".

Otra característica que presenta este tipo de litigios es que en el caso que un Grupo Especial o el Órgano de Apelación considere que la medida que no sea contraria a los acuerdos abarcados anula o menoscaba una ventaja resultante del acuerdo, o compromete el logro de alguno de sus objetivos, no podrá obligar a revocar la medida, sino que recomendarán que el miembro otorgue un ajuste mutuamente satisfactorio ${ }^{56}$.

En la primera ocasión que un panel se vio enfrentado a esta cuestión, observó que la norma del artículo XXIII tiene por objetivo fundamental proteger el equilibrio de las concesiones arancelarias. De esta manera, la idea que subyace a las reclamaciones sin infracción es que una concesión arancelaria puede verse frustrada no solo por medidas contrarias al Acuerdo General, sino que también por medidas compatibles con este. "Para animar a las partes contratantes a otorgar concesiones arancelarias debe por tanto concedérseles el derecho a buscar resarcimiento cuando otra parte contratante menoscabe una concesión recíproca a consecuencia de la aplicación de cualquier medida compatible o no con el Acuerdo General" 57 . A mayor abundamiento, agregó que en caso contrario "no tendrían interés en otorgar concesiones arancelarias y el Acuerdo General dejaría de tener utilidad en tanto que marco jurídico para la incorporación de los resultados de las negociaciones comerciales" 58 .

Luego en el asunto Japón - Películas ${ }^{59}$, un Grupo Especial consideró que la acción es un instrumento excepcional de la solución de diferencias, debido a que "los miembros negocian las normas que convienen en seguir y solo excepcionalmente prevén la posibilidad de que se dirijan contra ellas impugnaciones por medidas que no infringen esas normas" 60 .

\footnotetext{
56 Artículo 26.1 b) del ESD.

57 Comunidad Económica Europea - Primas y Subvenciones Abonadas a los Elaboradores y a los Productores de Semillas Oleaginosas y Proteínas Conexas destinadas a la Alimentación Animal (Comunidad Económica Europea - Semillas Oleaginosas), IBDD 37s/93 - L/6627, Informe del Grupo Especial adoptado el 25 de enero de 1990, párrafo 144.

58 Comunidad Económica Europea - Semillas Oleaginosas, párrafo 148.

59 Japón - Medidas que Afectan a las Películas y el Papel Fotográfico de Consumo (Japón Películas), WT/DS44/R, Informe del Grupo Especial de 31 de marzo de 1998, párrafos 10.33 y ss.

60 Japón - Películas, párrafo 10.36. Los comentarios a esta sentencia, así como el tratamiento de las reclamaciones sin infracción pueden verse en CHO, S - Oroidismon (1998) "GATT
} 
De acuerdo al Grupo Especial, el artículo XXIII.1 b) del GATT contiene tres elementos que la parte reclamante debe acreditar para formular su demanda, a) la aplicación de una medida por un miembro de la OMC, b) una ventaja resultante del acuerdo pertinente, y c) anulación o menoscabo de la ventaja resultante por aplicación de dicha medida ${ }^{61}$.

En cuanto a la aplicación de una medida, el Grupo Especial consideró que la expresión "medidas" a los efectos del artículo XXIII.1 b) tiene un alcance amplio, pudiendo abarcar tanto las leyes o reglamentos publicados por un gobierno, así como actos de gobierno que, no siendo vinculantes, tenga efectos similares a los de una medida vinculante ${ }^{62}$. Tampoco descartó que una acción realizada por particulares pueda considerarse una medida gubernamental "si hay una intervención del gobierno suficiente en ella"63.

En relación con las ventajas resultantes del acuerdo pertinente, se suele entender como la expectativa legítima de mejores oportunidades de acceso a los mercados derivada de las concesiones arancelarias correspondientes. A su vez, para que las expectativas sean legítimas se deben tener en cuenta todas las medidas de la parte que haga la concesión que podrían haberse previsto razonablemente en el momento de la concesión $^{64}$. A su vez, las ventajas resultantes de concesiones otorgadas en rondas sucesivas de negociaciones arancelarias pueden generar de forma autónoma expectativas razonables de mejoramiento de acceso a los mercados $^{65}$.

Por último, para acreditar la relación causal, el Grupo Especial señaló que se debe "demostrar que la competitividad de los productos importados objeto de una concesión (arancelaria) pertinente en materia de acceso a los mercados se halla trastornada por ("anulada o menoscabada [...] a consecuencia de") la aplicación de una medida que no ha sido prevista razonablemente" 66 .

Non - Violation Issues in the WTO Framework: Are They Achilles' Heel of the Dispute Settlement Process?", Harvard International Law Journal, vol. 39, ํㅜ 2, pp. 311 y ss.

Japón - Películas, párrafo 10.41.

Japón - Películas, párrafo 10.49.

Japón - Películas, párrafo 10.56 .

Japón - Películas, párrafo 10.61 .

Ello en contra de la tesis del Japón, que argumentaba que las expectativas razonables debían limitarse a los resultados de la Ronda Uruguay (Japón - Películas, párrafo 10.66).

66 Este hecho debe ser acreditado por la parte reclamante, a diferencia del hecho que la medida haya sido prevista razonablemente, cuestión que se presume a favor de esta (Japón - Películas, párrafos 10.79 y 10.82). 


\section{5) PARTES Y TERCEROS EN EL PROCESO}

Solo los Estados miembros de la OMC pueden actuar en el SSD, ya sea como partes, así como también en calidad de terceros en la diferencia. Ni la Secretaría de la OMC, ni los Estados observadores, ni otras organizaciones internacionales, así como tampoco gobiernos regionales ni locales de Estados miembros pueden iniciar procedimientos en el MSD de la OMC. Tampoco tienen acceso directo los privados, ya sea las ONG, personas individuales o empresas, ni aun públicas de un Estado miembro, aun cuando ellos suelen ser los más afectados frente a las posibles violaciones de las normas de la OMC por parte de otro Estado miembro, o tiene un mayor interés en que una medida sea retirada por ser contraria al derecho $\mathrm{OMC}^{67}$. Sin embargo, la práctica de los Grupos Especiales ha permitido la introducción limitada de observaciones a través de las denominadas comunicaciones Amicus Curiae, que analizaremos a continuación.

\section{(5.1.) LOS TERCEROS EN EL PROCESO}

El artículo 10 del ESD consagra el derecho de los Estados miembros de la OMC de participar como terceros Estados en la controversia ante los Grupos Especiales y el Órgano de Apelación ${ }^{68}$.

Para poder ejercer este derecho se requiere primeramente ser Estado miembro de la OMC. Ningún Estado que no sea miembro tiene derecho a participar en esta calidad ante el SSD. En segundo lugar, se requiere que tenga un interés sustancial en la controversia, y que así lo haya notificado al OSD. Con esas constataciones, el Estado tercero en la controversia puede ser oído ante el Grupo Especial y puede presentar a este comunicaciones por escrito, así como también se le dará traslado de las comunicaciones que presenten las partes en la controversia al Grupo Especial antes de su primera reunión. Por su parte, dichas comunicaciones deben verse reflejadas en el informe ante el Grupo Especial ${ }^{69}$.

$67 \quad$ En este sentido puede verse KESSIE, E. (2000). "Enhancing Security and Predictability for Private Business Operators under the Dispute Settlement System of the WTO", Journal of World Trade, vol. $34 \mathrm{~N}^{\circ}$ 6, pp. 1 y ss.

68 Sobre la participación de terceros Estados en las controversias en el GATT, así como las particularidades de esta participación en relación con el derecho internacional público, puede verse Covelli N. (1999). "Public International Law and Third Party Participation in WTO Panel Proceedings", Journal of World Trade, vol. $33 \mathrm{~N}^{\circ} 2$, pp. 125 y ss.

69 Artículo 10.2 y 10.3 del ESD. El Órgano de Apelación ha entendido que la exigencia de dar traslado a los terceros en aplicación del artículo 10.3 del ESD es amplio, abarcando todas las comunicaciones que presentaren las partes antes de la primera reunión, aun cuando fuere la única reunión del Grupo Especial (Estados Unidos - Trato Fiscal Aplicado a las Empresas de Venta en el Extranjero (WT/DS108/AB/RW), Informe del Órgano de Apelación sobre el Recurso de las Comunidades Europeas al párrafo 5 del artículo 21 del ESD, de fecha 14 de enero de 2002, párrafo 251). 
Los Grupos Especiales, sin embargo, pueden conceder "derecho más amplios" a los terceros participantes en la controversia, aunque ello queda a la razonable discreción y autoridad de los mismos. Esta discreción y autoridad, sin embargo, "no son ilimitadas y, por ejemplo, están circunscritas por los requisitos que impone el respeto de las garantías de procedimientos debidas" 70 , así como también por los derechos garantizados a los terceros por las disposiciones del $\mathrm{ESD}^{71}$.

Los terceros, por su parte, no pueden formular alegaciones ${ }^{72}$, así como tampoco tienen derecho a recurrir al Órgano de Apelación en el caso que el informe no satisfaga sus intereses. Sin embargo, durante el procedimiento de apelación pueden presentar comunicaciones, así como también el Órgano de Apelación puede darles la oportunidad de ser oídos. En todo caso, para poder actuar en apelación deben haber notificado el interés sustancial ante el OSD durante el transcurso del procedimiento ante el Grupo Especial, perdiendo la posibilidad de comparecer solo ante el Órgano de Apelación ${ }^{73}$.

\section{(5.2.) LAS COMUNICACIONES AMICUS CURIAE}

Los Grupos Especiales y el Órgano de Apelación pueden aceptar y considerar en su argumentación las denominadas comunicaciones amicus curiae $^{74}$. Estas comunicaciones suelen provenir de Organizaciones No Gubernamentales, incluidas en ellas asociaciones de industriales o escritos provenientes de académicos.

La facultad de recibir este tipo de escritos se infiere de la disposición del artículo 13 del ESD, que señala que "Cada Grupo Especial tendrá el derecho de recabar información y asesoramiento técnico de cualquier persona o entidad que estime conveniente", así como también de los acuerdos a que pueda llegar el Grupo Especial de conformidad al artículo 12.1.

En el caso Estados Unidos - Camarones, el Órgano de Apelación señaló que el párrafo 1 del artículo 12 ESD "autoriza a los grupos especiales a no seguir o a complementar los Procedimientos de Trabajo que se recogen en el Apéndice 3 del ESD e incluso a elaborar sus propios procedimien-

\footnotetext{
70 Estados Unidos - Ley Antidumping de 1916, WT/DS136/AB/R y WT/DS162/AB/R, Informe del Órgano de Apelación de 28 de agosto de 2000, párrafo 150.

71 Estados Unidos - Trato Fiscal Aplicado a las Empresas de Venta en el Extranjero WT/ DS108/AB/RW, Informe del Órgano de Apelación sobre el Recurso de las Comunidades Europeas al párrafo 5 del artículo 21 del ESD, de fecha 14 de enero de 2002, párrafo 243.

72 Chile - Sistema de Bandas de Precios y Medidas de Salvaguardia Aplicados a Determinados Productos Agrícolas, WT/DS207/AB/R, Informe del Órgano de Apelación de 23 de septiembre de 2002, párrafo 163.

73 Artículo 17.4 del ESD.

74 En inglés también se les conoce como friends of the court.
} 
tos de trabajo, tras consultar a las partes en la diferencia"75, de manera que confiere "facultades amplias y extensas para emprender y controlar el proceso mediante el cual recaban información"76. De esta manera, los Grupos Especiales pueden recibir y aceptar comunicaciones amicus curiae con independencia que la entidad que emite la comunicación haya sido requerida por el Grupo Especial para tal efecto ${ }^{77}$.

En el caso de la recepción de comunicaciones amicus curiae en la etapa de apelación, en el asunto Comunidades Europeas - Amianto, el Órgano de Apelación dispuso de un procedimiento especial para la autorización y recepción de comunicaciones amicus curiae, por parte de Organizaciones No Gubernamentales, estableciendo criterios específicos que debían tener las solicitudes de comunicación, así como los criterios para tener estos en cuenta o no ${ }^{78}$.

También se pueden recibir comunicaciones amicus curiae de parte de Estados miembros de la OMC que no actúen como parte ni como terceros en la controversia. En el asunto Comunidades Europeas - Sardinas, el Órgano de Apelación señaló que del hecho que tenga la facultad de recibir escritos amicus curiae de privado se sigue, a fortiori, que también tiene la facultad de recibirlos de Estados miembros de la OMC ${ }^{79}$.

El problema que se planteaba en este caso, es que la aceptación de este tipo de comunicaciones de otros Estados miembros que no participan ni como parte ni como tercero en la controversia implicaría poner en pie de desigualdad a quienes han reservado efectivamente sus derechos como terceros en el procedimiento. Sin embargo, el Órgano de Apelación desechó este argumento, constatando que quienes hayan participado como terceros ante el Grupo Especial tienen el derecho de presentar en apelación una comunicación escrita. Por contrapartida, el Órgano de Apelación tiene la obligación de examinarla. En el caso de los Estados miembros que presenten escritos amicus curiae en la etapa de apelación no tiene garantizado que el Órgano de Apelación acepte y examine el escrito, y tampoco tiene derecho a asistir a la audiencia en ninguna cali$\mathrm{dad}^{80}$.

\footnotetext{
75 Estados Unidos - Prohibición de las Importaciones de Determinados Camarones y Productos del Camarón (Estados Unidos - Camarones), WT/DS58/AB/R, Informe del Órgano de Apelación de 12 de octubre de 1998, párrafo 105.

76 Estados Unidos - Camarones, párrafo 106.

77 Estados Unidos - Camarones, párrafo 108.

78 Comunidades Europeas - Medidas que Afectan al Amianto y a los Productos que Contienen Amianto (Comunidades Europeas - Amianto), WT/DS135/AB/R, Informe del Órgano de Apelación de 12 de marzo de 2001, párrafos 50 y ss., especialmente el párrafo 52.

79 Comunidades Europeas - Denominación Comercial de Sardinas (Comunidades Europeas - Sardinas), WT/DS231/AB/R, Informe del Órgano de Apelación de 26 de septiembre de 2002, párrafo 164 .

80 Comunidades Europeas - Sardinas, nota 69.
} 
Entendemos que esta facultad que asiste a quienes no pueden ser parte en la controversia ante los Grupos Especiales y el Órgano de Apelación se ha constituido en una poderosa herramienta de legitimidad y transparencia del propio sistema de solución de diferencias, que ha permitido la participación de la sociedad civil al menos en parte del proceso de decisiones del OSD, influyendo a la vez un mayor desarrollo de la calidad del precedente del propio sistema, como una forma de asistir en una mayor adecuación de las conductas del Estado en el sistema multilateral de comercio. Sin embargo, entendemos que el concepto de legitimidad y transparencia habrán de ser reforzados en el sistema multilateral de comercio, lo que invita a plantearse un reforzamiento del acceso de comunicaciones amicus curiae al sistema.

\section{CONCLUSIONES}

El reforzamiento del MSD en la Ronda Uruguay de Negociaciones Comerciales ha otorgado al sistema multilateral de comercio un elemento importante de destacar en materia de seguridad jurídica y previsibilidad en el comportamiento de los Estados dentro de sus normas.

Ello se demuestra en la regla del consenso negativo en la aprobación de los informes, que ha permitido otorgar una mayor seguridad en las propias actuaciones del Órgano de Apelación, que le permite interpretar con un alto grado de libertad las normas de los acuerdos abarcados. Este grado de libertad, a su vez, ha permitido dar un giro al SSD desde un sistema con orientaciones políticas hacia un sistema con orientación normativa, permitiendo unificar criterios futuros de actuación de los Estados miembros.

No es el objetivo del sistema sancionar a los Estados ni tampoco resolver la controversia, sino que solo pretende lograr una solución mutuamente satisfactoria para las partes en la diferencia, así como también la supresión de medidas que puedan considerarse incompatibles con las normas de la OMC, en el caso que el primer objetivo no sea cumplido.

Desde esta perspectiva, el éxito que ha demostrado el SSD durante los quince años de existencia ha constituido también un soporte extraordinariamente relevante para el propio sistema multilateral de comercio, que ha visto en el Órgano de Apelación un verdadero apoyo para la cooperación en materia comercial. De esta manera, las obligaciones no solo pueden identificarse en las normas de los acuerdos OMC, sino que además su desarrollo e interpretación puede apreciarse en la actuación de los Grupos Especiales y del Órgano de Apelación, los que han definido con mayor precisión la conducta de los Estados miembros. Si bien los informes adoptados son solo obligatorios para las partes en la diferencia, los criterios subyacentes son de conocimiento necesario para los miembros 
no partes en la diferencia, de manera de establecer con mayor claridad el alcance de sus obligaciones.

Uno de los aspectos procesales relevantes a tener presente de cara a una eventual reforma o un nuevo reforzamiento del SSD en la actual Ronda de Doha de Negociaciones Comerciales, dice relación con las comunicaciones amicus curiae, como un elemento de mayor legitimidad y transparencia del sistema hacia la opinión pública mundial.

\section{BIBLIOGRAFÍA}

- Abi-SaAb, G. (2006). "The Appellate Body and Treaty Interpretation", en Sacerdoti, G. - Yanovich, A. - Bohanes, J. (eds.): The WTO at Ten. The Contribution of the Dispute Settlement System, Cambridge University Press, pp. 453-464.

- Bou Franch (2005). Nuevas Controversias Internacionales y Nuevos Mecanismos de Solución, Valencia: Tirant lo Blanch, 588 pp.

- Cho, S - J. (1998). "GATT Non-Violation Issues in the WTO Framework: Are They Achilles' Heel of the Dispute Settlement Process?", Harvard International Law Journal, vol. 39, N 2, pp. 311-351.

- Covelli N. (1999). "Public International Law and Third Party Participation in WTO Panel Proceedings", Journal of World Trade, vol. $33 \mathrm{~N}^{\circ}$ 2, pp. 125-139.

- Diez-Hochleitner, J. (2005). "La Unión Europea ante la Reforma del Sistema de Solución de Diferencias de la OMC", en De Faramiñan, J. M. (coord.): Globalización y Comercio Internacional: Actas de la XX Jornadas de la Asociación Española de Profesores de Derecho Internacional y Relaciones Internacionales, Madrid, Asociación Española de Profesores de Derecho Internacional y Relaciones Internacionales - BOE, 2005, pp. 61-88.

- Fernández, X. (2006). La OMC y el Derecho Internacional, Madrid, Marcial Pons, 603 pp.

- Footer, M. (2001). "Developing Country Practice in the Matter of WTO Dispute Settlement", Journal of World Trade, vol. 35, No 1, pp. 55-98.

- González, L. N. (1998). Política Comercial y Relaciones Exteriores de la Unión Europea, Madrid: Tecnos, 431 pp.

- Hudec, R. (1990). The GATT Legal System and World Trade Diplomacy, New Hampshire: Butterworth Legal Publishers, 376 pp.

- Jackson, J. H. (2000). The Jurisprudence of GATT \& the WTO, Cambridge University Press, 2000, 497 pp.

- Kessie, E. (2000). "Enhancing Security and Predictability for Private Business Operators under the Dispute Settlement System of the WTO”, Journal of World Trade, vol. 34 N$^{\circ}$ 6, pp. 1-17. 
- McGovern, E. (1996). "Dispute Settlement in the GATT - Adjudication or Negotiation?", en Hilf, M., Jacobs, F. and Petersmann, E. - U. (eds.): The European Community and GATT, Deventer: Kluwer Law and Taxation Publishers, pp. 73-84.

- Mitchell, A. (2008). Legal Principles in WTO Disputes, Cambridge University Press, 308 pp.

- Montañá I Mora, M. (1997). La OMC y el Reforzamiento del Sistema GATT, Madrid: McGraw-Hill, 230 pp.

- Nichols, P. (1996). "GATT Doctrine", Virginia Journal of International Law, vol. 36, No 2, pp. 379-466.

- Oesch, M. (2005). Standards of Review in WTO Dispute Resolution, Oxford University Press, $276 \mathrm{pp}$.

- Pauwelyn, J. (2001). "The Role of Public International Law in the WTO: How far can we go?”, American Journal of International Law, vol. 95 , pp. 535-578.

- Petersmann, E.-U. (1994). "The Dispute Settlement System of the World Trade Organization and the Evolution of the GATT Dispute Settlement System Since 1948”, Common Market Law Review, vol. 31, No 6, pp. 1157-1244.

- Petersmann, E.-U. (1997). International Trade Law and the GATT/ WTO Dispute Settlement System, London: Kluwer Law International, $688 \mathrm{pp}$.

- Qureshi, A. (1999). International Economic Law, London: Sweet \& Maxwell, 417 pp.

- Rodríguez, M. (2005). "La Solución de Controversias en la OMC. Evolución y Reforma (1995-2004)", en De Faramiñan, J. M. (coord.): Globalización y Comercio Internacional: Actas de la XX Jornadas de la Asociación Española de Profesores de Derecho Internacional y Relaciones Internacionales, Madrid, Asociación Española de Profesores de Derecho Internacional y Relaciones Internacionales - BOE, pp. 43-60.

- Stewart, T. (ed.) (1993). The GATT Uruguay Round. A Negotiating History (1986-1992), The Hague: Kluwer Law International, 2878 pp.

- Van Damme, I. (2009). Treaty Interpretation by the WTO Appellate Body, Oxford University Press, 419 pp.

- World Trade Organization (2004). A Handbook on the WTO Dispute Settlement System, Cambridge University Press, 215 pp.

\section{Casos}

- Argentina - Medidas que afectan a las Importaciones de Calzados, Textiles, Prendas de Vestir y otros Artículos (Argentina - Calzados), 
WT/DS56/AB/R, Informe del Órgano de Apelación de 27 de marzo de 1998.

- Chile - Sistema de Bandas de Precios y Medidas de Salvaguardia Aplicados a Determinados Productos Agricolas, WT/DS207/AB/R, Informe del Órgano de Apelación de 23 de septiembre de 2002.

- Comunidad Económica Europea - Primas y Subvenciones Abonadas a los Elaboradores y a los Productores de Semillas Oleaginosas y Proteinas Conexas destinadas a la Alimentación Animal, IBDD 37s/93 L/6627, Informe del Grupo Especial adoptado el 25 de enero de 1990.

- Comunidades Europeas - Medidas que Afectan al Amianto y a los Productos que Contienen Amianto, WT/DS135/AB/R, Informe del Órgano de Apelación de 12 de marzo de 2001.

- Comunidades Europeas - Denominación Comercial de Sardinas, WT/DS231/AB/R, Informe del Órgano de Apelación de 26 de septiembre de 2002.

- Comunidades Europeas - Subvenciones a la Exportación de Azúcar, WT/DS265/AB/R, WT/DS266/AB/R y WT/DS283/AB/R, Informe del Órgano de Apelación de 28 de abril de 2005.

- Estados Unidos - Pautas para la Gasolina Reformulada y Convencional, WT/DS2/AB/R, Informe del Órgano de Apelación de 29 de abril de 1996.

- Estados Unidos - Medida que Afecta a las Importaciones de Camisas y Blusas de Tejidos de Lana Procedentes de la India, WT/DS33/AB/R, Informe del Órgano de Apelación de 25 de abril de 1997.

- Estados Unidos - Prohibición de las Importaciones de Determinados Camarones y Productos del Camarón, WT/DS58/AB/R, Informe del Órgano de Apelación de 12 de octubre de 1998.

- Estados Unidos - Ley Antidumping de 1916 (WT/DS136/AB/R y WT/DS162/AB/R), Informe del Órgano de Apelación de 28 de agosto de 2000.

- Estados Unidos - Trato Fiscal Aplicado a las Empresas de Venta en el Extranjero (WT/DS108/AB/RW), Informe del Organo de Apelación sobre el Recurso de las comunidades Europeas al párrafo 5 del artículo 21 del ESD, de fecha 14 de enero de 2002.

- Estados Unidos - Examen por Extinción de los Derechos Antidumping sobre los Productos Planos de Acero al Carbono Resistentes a la Corrosión procedentes del Japón, WT/DS244/AB/R, Informe del Órgano de Apelación de 15 de diciembre de 2003.

- Estados Unidos - Subvenciones al Algodón Americano (Upland), WT/ DS267/AB/R, Informe del Órgano de Apelación de 3 de marzo de 2005.

- Japón - Impuestos sobre las Bebidas Alcohólicas, WT/DS8/AB/R, Informe del Órgano de Apelación de 4 de octubre de 1996. 
- Japón - Medidas que Afectan a las Películas y el Papel Fotográfico de Consumo, WT/DS44/R, Informe del Grupo Especial de 31 de marzo de 1998.

- Méjico - Medidas Fiscales sobre los Refrescos y otras Bebidas (Méjico Refrescos), WT/DS308/AB/R, Informe del Organo de Apelación de 6 de marzo de 2006. 